\title{
Factors that Influence Practice-Nurses to Promote Physical Activity
}

\author{
Article by Sophonie Ndahayo \\ Ph.D in Public Health, Texila American University, India \\ E-mail:monsniyo@gmail.com
}

\section{Source}

McDowell, N., McKenna, J., and Naylor, P-J. (1997). Factors that influence practicenurses to promote physical activity. British Journal of Sports Medicine volume 31 number 4, pages 308-313.

\section{Introduction}

McDowell, McKenna, and Naylor (1997) research article entitled "Factors that influence practice nurses to promote physical activity", which appeared in the British Journal of Sports Medicine number 31, pages 308-313 had as objective to investigate what factors may influence practice-nurses to promote physical activity. The study is of paramount importance because there is a worldwide concern about increasing rates of obesity and decreasing population levels of physical activity. Yet, it has been argued that primary healthcare professionals are ideally placed to promote physical activity within local communities (Douglas et al 2006).Though it has been argued that primary healthcare professionals are ideally placed to promote physical activity within local communities (Douglas et al 2006), Steptoe et al. (1999) stated that in Catalonia in the United Kingdom, there was a lack of evidence regarding the levels of physical activity promotion. There are various constraints that impede the success of physical activity promotion programmes. Nevertheless, this study showed that the two stage measures (activity promotion and personal behaviour) of the health care professional are associated with important differences in patient and practice factors for physical activity promotion. Their view will be presented in the following order: summary of the article; structure; critique that will focus on: the authority of the authors/ researchers, accuracy of style of writing, relevance of the study subject or topic, objectivity of the research and its stability. Finally, the review will analyze the graph and draw a conclusion on the whole article.

\section{Review of literature}

The Health Education Authority (1992) stipulated that recent evidence suggested that British adults do not participate in sufficient physical activity to offset health problems. Recently, Donaldson (2009) stated that the benefits of regular physical activity to health, longevity, well-being and protection from serious illness have long been established. They easily surpass the effectiveness of any drugs or other medical treatment. The challenge for everyone, young and old alike, is to build these benefits into their daily lives. If a medication existed which had a similar effect, it would be regarded as a "wonder drug" or "miracle cure". Donaldson (2009) concluded that it is crucial to encourage a culture of physical fitness in the population which spans all ages. However, (Hillsdon \& Thorogood 1992) had noted that the challenge remained to identify efficient strategies that encourage, even modest, increases of physical activity in large sections of the public. According to the United Kingdom (UK): Department of Health (1992)and the General Medical Services (1993) one possible means to increase levels of physical activity in the UK is the government supported concept of preventative care in which general practitioners (GPs) are encouraged to offer regular health checks to their adult patients, and health promotion services to all registered patients. These facts have been supported by Douglas et al 2006 whose findings revealed that there were high levels of enthusiasm for physical activity promotion amongst health visitors and practice nurses. This is in line with the World Health Organisation's 2008-2013 action plan for global strategy for the prevention and control of non-communicable whose one of the objectives 
Texila International Journal of Public Health

Volume 5, Issue 1, Mar 2017

states: to promote interventions to reduce the main shared modifiable risk factors for noncommunicable diseases including unhealthy diets and physical activity. It is noteworthy that there are various barriers to effective PA promotion that include patients', health-provider and system barriers. The review of literature evaluated the quality and the meaning of the studies done before, and arrived at a conclusion about the problem based on the studies evaluated. It has shown the gap in knowledge which prompted the research. The literature was relevant, up-to-date and coherent.

\section{Article summary}

The reviewed article, McDowell, McKenna, and Naylor (1997) investigated factors that may influence practice nurses to promote physical activity (PA) in Avon in England. The problem has been that England is faced with diseases of affluence that can be alleviated by regular PA. However, research has shown that Britons do not participate in sufficient PA to offset health problems. Though general practice-based (GPs) PA promotion has been found ideal, GPs are overwhelmed by workloads and various barriers. Actually, PA promotion is carried out by Practice Nurses (PNs), whose knowledge and skills are uncertain, which justified the study. A questionnaire was sent to all PNs $(n=272)$, with $72.1 \%$ return rate after data cleaning. Data were collected under personal, patient status, and practice fact factors respectively. Data were analyzed statistically using a Mann-Whitney test at $0.05 \%$ level of significance. Analyzing results based on the trans-theoretical model appeared difficult, a further analysis was done using a dichotomized stage: promotion behaviour and own activity. Results showed that a large proportion of PNs are promoting PA. Furthermore, data revealed that PNs who are active themselves promoted regularly PA and perceived system barriers as of less limiting effects on their level of PA promotion. There was a significant difference in the hours of training that the promoting and restricted promoting PNs had received in the past five years. It is noteworthy that lack of time was limiting for all PNs. According to authors, findings may illustrate behaviours and perceptions of 1994 - year of data collection.

\section{Article structure}

With the page number in top left corner, name of the publishing journal in the top-right corner, the topic centred a little bit lower the above, followed by the name of the authors, the article started with an abstract, which gave the objective, methods, results, and the conclusions. Full addresses of the authors, date the article was accepted by the journal are indicated on the first page. The methods followed the systematic way of research; however, there was no mention of how ethical considerations were followed. Results section highlighted the data collection return rate. The proportion of respondents who promote physical activity and the level of significance of the statistic test were also stipulated. Though the article was written in paragraph form, starting by a tab, which facilitated the sequence of ideas, some paragraphs were too long, thwarting the benefit of the shorter ones. It is noteworthy that the use of sub-headings under methods allowed getting the flow of the description of data collection, analysis and results. However, the concept was abandoned in the discussion, which made reading appear tiring, though paragraphing lessened the effects. The conclusions suggested factors contributing to PA promotion and what further investigations could be done. The section was short, precise and to the point. Though the article does not have an acknowledgements' section, thanks were expressed to Pat Turton of Avon Health and all respondents. The references were given and appeared up-to-date. However, following the American Psychological Association guidelines - references appear in alphabetical order could have been the best option. The article structure was reasonably developed and accessible in PDF, which improves its viral spoilage on the worldwide web. The publishing journal, the topic and authors' names appear on the right-hand side with page numbers indicated alternating on left and right-top side of each page. 


\section{Article critique}

\section{Authority}

The article was published in the British Journal of Sports Medicine, which was established in 1964. It is a peer-reviewed medical journal that publishes the latest advances in clinical practice and research in all aspects of sports medicine. It is an official journal of the British Association of Sports and Exercise Medicine. The journal covers scholarly work under the coordination of Professor Karim Khan, a medical doctor, FASCM and PhD as editor-in-chief.

The author's credibility was established through their professional qualification and a number of publications in the subject area covered by the article reviewed. The authors are professionals in the schools of physiotherapy and occupational therapy and that of exercise and health resource unity, as well as in the Ministry of health in Britain, which shows the credibility of both the journal and the authors of the article. Finally, up-to-date references crowned the authority at hand.

\section{Accuracy}

Accuracy or precision is their quality of being true or correct. The article contained information from then current research findings. All important facts advances by the researchers had supported from empirical evidence-based sources. The literature review presented in the article was from recent sources from scientific research articles, many of which were from peer-reviewed journals. The conclusions were derived from the data. However, the precision in measurements and analyses could be improved through use of a parametric statistical test since the sample was not small, and there was no comparison of magnitude was to be done. Furthermore, parametric could have added the generalizability of conclusions. Factors could be grouped together with respective loadings, which could clearly show the effects on the studied population. Nevertheless, considering the caliber of the editorial and peer-review process the article went through, it is safe to say that the article's accuracy met the standards.

\section{Currency}

At the time of publication, the article was current and used current literature. However, the reader must take note of the delay in publication. The article reported that the data of this study were collected in 1994 and illustrates behaviours and perceptions related to that time period according to the authors. It was accepted for publication in July 1997. The year of publication of the references ranged from 1992 to 1996. Only one source seemed old, which was 1989. The content and the topic were also current as problems of alleviating physical inactivity health effects were and still issues on public health and clinical medicine.

\section{Relevance}

The article was relevant. It had a scientific relevance in that scientists benefited from its findings which elucidated factors may influence practice nurses to promote physical activity in the studied population. This was very beneficial as scientists faced a challenge of identify efficient strategies that would encourage increases of physical activity in large sections of the public. Furthermore, the article had a societal relevance in that it addressed a problem that affected the target population, even the whole nation. A solution to the problem would bring relief to the studied population through prevention and control of diseases of affluence. Lastly, the solution would contribute to the development of the society. Finally, results of the research helped in preparation of the curriculum for future practice nurses, which added the educational relevance. Therefore, the article was very relevant.

\section{Objectivity}

The objectivity of the article was demonstrated through statement of the aim of the research, which guided the process. There was elimination of researchers' bias through the use of questionnaire to collect data - respondents freely filled-in the tool. In addition, 
Texila International Journal of Public Health

Volume 5, Issue 1, Mar 2017

scientific measurements were used through data cleaning - exclusion of tools with incomplete information. The use of statistical data analysis stating the level of significance of the test also demonstrated the objectivity of the article. Furthermore, there was balance in selection of source material. Finally, the researchers mentioned the limitations of the findings - uneven distribution for stage measures, which made them adopt dichotomized stages (Fig 2 in the article). It was also mentioned that results could illustrate behaviours and perceptions of the year 1994, when data were collected, which is a good indicator of objectivity of the article.

\section{Stability}

The article, with its source in a reputable academic journal, which is run by reliable academic researchers, is a stable source of information. In addition, the authors used evidence-based literature to support their both the justification of the problem and discuss their findings.

\section{Analysis of graph/image/table}

The article has two tables, and two figures. Table one contains the stage of change and statements thereof. It is well-labeled with a title which included the name of the variable. Columns are mutually exclusive, but the rows could have been indicated by lines to improve readability. Table two has: ssummary of patient, personal, and practice factors by dichotomised stage measures as a valid title and has a number. Neither columns nor rows are mutually exclusive, something which could simplify readability. It is worth mentioning that frequencies, percentages and totals were included in the tables. Figures were well-labelled and allocated numbers. Variables are indicated and one can easily decipher the information contained in the figure.

\section{Recent advances related to the topic}

General practice is an important setting for promoting physical activity (PA) given the great population-wide access to people of all ages. Health-care providers are seen by the public as credible sources of information, and patients perceive health promotion to be part of the general practice's role, including physical exercise promotion (nphp, 2002).However, the World Health Organisation (WHO) recognises that health promotion requires a comprehensive and lifespan approach across different groups and settings, and is enhanced through partnership across multiple sectors. The WHO guidelines are validated by the findings of Ribera, McKenna and Riddoch (2005): physical activity promotion was opportunistic, focused on selected patients, highly dependent on personal activity interests. Health policy should focus on integrating PA promotion into more practice consultations. Nevertheless, the Royal College of Nursing (RCN) (2012) argued that nurses have a key role in minimising the impact of illness, promoting health and function, and helping people maintain their roles at home, at work, at leisure and in their communities... nurses are in a unique position to contribute to the lifespan approach to health protection and health improvement. Every interaction should be seen as an opportunity to promote health and prevent illness. RCN has come up with innovation in upstream nursing with good practice grouped under prevention, protection and promotion, which should include PA promotion.

Furthermore, results of Douglas et al. (2006) stated that 90\% $(\mathrm{n}=149)$ of health visitors and $88 \%(\mathrm{n}=186)$ of practice nurses said that they were very likely or likely to recommend all apparently healthy adult patients to take moderate exercise. However, Huijg et al. (2013) seems to argue against saying that introduction of efficacious physical activity interventions in primary health care is a complex process. Within primary health care practice, rates of PA promotion are suboptimal (Glasgow et al 2001 \& Fallon, Wilcox, \& Laken 2006). A conclusion was made that organizations and professionals need to make the decision to work with an intervention -adoption, deliver it as intended- implementation, and continue to use it over a longer period of time - continuation (Durlak \& DuPre 2008). 


\section{Conclusion}

This article provides a valuable understanding into the physical activity promotion behaviours of practice nurses (PNs). The literature review showed the discrepancy in knowledge, which prompted the study. Due to attitudinal and system barriers, the duty of promoting physical activity (PA), which initially belong to general practitioners (GPs) has been delegated to PNs, whose effectiveness is unclear. Therefore, it has been pertinent to investigate the factors that may influence PNs to promote PA. Based on a reliable model stage of change model, the study was based on three elements: patient, practice and personal (provider) factors. Results showed that a large number of active PNs are promoting PA. Two clusters of barriers were identified; lack of time, measurable success, and lack of resources have the greatest effect. Contrary to previous studies (PA promotion depends on smaller number of patient lists), in this study, active PNs were PA promoting and rated lack of resources, protocols and success as of less limiting effect on PA promotion. Of the PNs who reported not promoting PA, $2 / 3$ had not received formal training in the past five years. Having followed the systematic research process, despite the limitations of the study, the article stands a high stability. However, suggestions would be: including males in the sample; changing the target population to patients as results were self-reports of PNs; encouraging PNs to avoid selective PA counselling; organising in-service- training to equip PNs with PA skills, and PNs and general practitioners' curriculum should include PA skills.

\section{References}

[1]. Calnan, M., Coyle, J. and Williams, S. (1994). Changing perceptions of general practitioner care. European Journal of Public Health vol 4, pp. 108-14.

[2]. Douglas, F., Teijliingen, V.E., Torrance, N., Fearn, P., Kerr, A. and Meloni, S. (2006). Promoting physical activity in primary care settings: health visitors' and practice nurses' views and experiences Journal of Advanced Nursing 55, (2) pp. 159-68.

[3]. Donaldson (2009). www.dh.gov.uk/publications

[4]. Department of Health. (1992). The Health of the Nation: a Strategy for Health in England. London: HMSO.

[5]. Douglas, F., Teijlingen, V. E., Torrence, N., Fearn, P., Kerr, A., and Meloni, S. (2006). Promoting physical activity in primary care settings: health visitors' and practice nurses' views and experiences. Journal of Advanced Nursing vol 55(2) pp. 159-68.

[6]. Durlak, J.A. and DuPre, E.P. (2008). Implementation matters: a review of research on the influence of implementation on program outcomes and the factors affecting implementation. American Journal of Community Psychology 41, pp. 327-350.

[7]. Fallon, E.A., Wilcox, S. and Laken, M. (2006). Health care provider advice for African American adults not meeting health behavior recommendations. Prev Chron Dis 3, pp. 1-12.

[8]. Florindo, A.A., Mielke, G.I., Gomes, G.A.d.O., Ramos, L.R., Bracco, M.M., Parra, D.C., Simoes, E.J., Lobelo, F. and Hallal, P.C. (2013). Physical activity counselling in primary health care in Brazil: a national study on prevalence and associated factors. BMC Public health 13, p. 794.

[9]. Glasgow, R.E, Eakin, E.G., Fisher, E.B., Bacak, S.J. and Brownson, R.C. (2001). Physician advice and support for physical activity: results from a national survey. American Journal of Preventive Medicine 21, pp. 189-196.

[10]. General Medical Services Committee. (1993). The New Health Promotion Package. London: British Medical Association.

[11]. Health Education Authority. (1992). Allied Dunbar National Fitness Survey. Ipswich: Ancient House.

[12]. Hillsdon, M., Thorogood, M. (1996). A systematic review of physical activity promotion strategies. Br_J Sports Med vol 30, pp. 84-89.

[13]. Huijg, J.M., Crone, M.R., Verheijden, M. W., Zouwe, N.v.d., Middelkoop, B. J.C. and Gebhardt, W. A. (2013). Factors influencing the adoption, implementation and continuation of physical activity intervention sin primary health care: a Delphi study. BMC Family Practice 14, p. 142. 
Texila International Journal of Public Health

Volume 5, Issue 1, Mar 2017

[14]. Steptoe, Doherty, Kendrick, Rink, and Hilton (1999). Attitudes to cardiovascular health promotion among general practitioners and practice nurses. Family practice 16 (2), 158-163.

[15]. www.who.int.

[16]. www.nphp.gov.au2002

[17]. www.ren.org.uk 